\title{
CONVERSION OF $A B$-INITIO FORCE FIELDS AND STRUCTURES TO MOLECULAR MECHANICS ENERGY FUNCTIONS
}

\author{
K. Palmö, L.-O. Pietilä and S. Krimm \\ Biophysics Research Division and Department of Physics, University of Michigan, Ann Arbor, \\ MI 48109, U.S.A.
}

(Received 30 May 1990; received for publication 7 February 1991)

\begin{abstract}
The rapid development of computers in recent years has brought increasingly complex compounds into the range of high level $a b$-initio calculations. Such calculations produce valuable results which in many cases would be difficult or even impossible to obtain, with comparable accuracy, in any other way (Fogarasi \& Pulay, Annu. Rev. Phys. Chem. 35, 191, 1984). Thus, it is highly desirable to be able to utilize these results in the construction of potential energy functions used in molecular mechanics (MM), molecular dynamics and Monte-Carlo calculations. For instance, the significance of quadratic cross terms in MM energy functions is still insufficiently explored (Lii \& Allinger, J. Am. Chem. Soc, 111, 8566, 1989), In order to make possible the complete utilization of $a b$-initio results in $\mathrm{MM}$ calculations, we have developed a method by which scaled $a b$-initio (or empirical) force fields and structures can be directly converted to $\mathrm{MM}$ potential energy parameters, without sacrificing any of the original accuracy with regard to vibrational frequencies or structure. Here we briefly outline the conversion procedure, a more complete analysis being published separately.
\end{abstract}

\section{INTRODUCTION}

In the molecular mechanics method (Pietilä, 1989) the potential energy function may be considered to consist of a quadratic part $V_{\mathrm{q}}$ and a nonquadratic part $V_{\text {nq }}$ :

$$
V=V_{\mathrm{u}}+V_{\text {nq }} \text {, }
$$

$V_{\text {nq }}$ accounts for van der Waals interactions, Coulombic interactions, periodic torsions etc while $V_{\mathrm{q}}$ is the potential energy arising from deformation of valence bonds and angles. Explicitly, $V_{\mathrm{q}}$ can be written:

$$
V_{\mathrm{q}}=\frac{1}{2} \sum_{i, j} F_{i j}^{\mathrm{MM}}\left(R_{i}-R_{00}\right)\left(R_{j}-R_{j 0}\right)
$$

where the $F_{i j} \mathrm{MM}_{\mathrm{S}}$ are called $\mathrm{MM}$ force constants, the $R_{i}$ S are valence coordinates and the parameters $R_{* 0}$ define the so-called reference (or intrinsic equilibrium) geometry. In the conversion from $a b$-initio we assume that $V_{\mathrm{ng}}$ is known and we only determine the MM force constants and the reference geometry. This is not a serious restriction, since the parameters of $V_{\mathrm{nq}}$ can be determined independently of those of $V_{q}$, e.g. by fitting to crystal properties and to $a b$-initio electrostatic potentials (Momany, 1978).

If the $a b$-initio force constant matrix is given in Cartesian coordinates as $\left(F_{k \alpha, 1 \beta}\right)$, where $k$ and $l$ run over the atoms and $\alpha, \beta=1,2,3$, the contribution of the nonquadratic interactions can be subtracted from it to yield a new matrix $\left(F_{k, 1 \beta}^{q}\right)$ that is purely associated with $V_{q}$. Using the chain rule, the second derivatives of $V_{\mathrm{q}}$ with respect to the internal coordinates, i.e. the MM force constants, are then found to be:

$$
\begin{aligned}
& F_{i j}^{\mathrm{MM}}=\sum_{k x}\left[\left(\frac{\partial V_{\mathrm{q}}}{\partial x_{\mathrm{k} \alpha}}\right)_{\bar{x}_{\mathrm{min}}}\left(\frac{\partial^{2} x_{k \alpha}}{\partial R_{i} \partial R_{j}}\right)_{\bar{K}_{\mathrm{man}}}\right. \\
& \left.+\sum_{l \beta}\left(\frac{\partial x_{k x}}{\partial R_{i}}\right)_{R_{11 \min }}\left(\frac{\partial x_{l p}}{\partial R_{j}}\right)_{R_{\min }} F_{k \alpha, l \beta}^{\mathrm{q}}\right],
\end{aligned}
$$

where $\bar{x}_{\min }$ and $\bar{R}_{\min }$ denote the minimum of the total potential $V$ in Cartesian and internal coordinates, respectively. In the expression above, the Cartesian gradient of $V_{\mathrm{q}}$ is readily obtained as the negative of the gradient of the nonquadratic interactions since, at the minimum, the total gradient is zero. The first and second derivatives of the Cartesians with respect to the (nonredundant) internal coordinates can be derived from the second derivatives of the internal coordinates with respect to the Cartesians by inversion of a power series (Arfken, 1970) and by requiring that the Cartesian displacement vectors do not represent any translation or rotation of the whole molecule.

To compute the reference geometry we take the derivative of equation (2) with respect to an internal coordinate $R_{n}$ and equate this to what is obtained for the same derivative using the chain rule, i.e.

$$
\sum_{j} F_{n j}^{\mathrm{MM}}\left(R_{j}-R_{i 0}\right)=\sum_{k \alpha}\left(\frac{\partial V_{\mathrm{q}}}{\partial x_{k \alpha}}\right)_{\bar{x}_{\min }}\left(\frac{\partial x_{k \alpha}}{\partial R_{n}}\right)_{\bar{R}_{\min }},
$$

where, for a nonlinear molecule composed of $\mathrm{N}$ atoms, the only unknowns are the $3 \mathrm{~N}-6 R_{j 0}$ 's. For $n=1, \ldots, 3 \mathrm{~N}-6$, equation (4) forms a set of $3 \mathrm{~N}-6$ linear equations which can be solved to yield the reference geometry $R_{f 0}, j=1, \ldots, 3 \mathrm{~N}-6$.

The conversion from ab-initio is mathematically exact so that the derived MM energy parameters yield 
Table 1. Some examples of ealoulated MM energy parameters for ethylene corresponding to van der Waals (vdW) interactions of different repulsive strengths"

\begin{tabular}{ccccccc}
\hline vdW & $\Delta R_{\mathrm{c}}(\boldsymbol{A})$ & $\begin{array}{c}\Delta \alpha \\
\text { (degrees) }\end{array}$ & $F\left(R_{c}\right)$ & $F(\alpha)$ & $F(\beta)$ & $F(\gamma)$ \\
\hline None & 0.000 & 0.0 & 1347 & 98 & 158 & 36 \\
$\times 1$ & 0.001 & -0.4 & 1341 & 97 & 152 & 35 \\
$\times 2$ & 0.005 & -2.3 & 1315 & 91 & 127 & 33 \\
$\times 4$ & 0.045 & -25.7 & 1214 & 66 & 30 & 21 \\
\hline
\end{tabular}

The first row corresponds to the original force field (no vdW interactions), vdW $\times 1$ means normal (realistic) $v d W$ interactions, $\mathrm{vdW} \times 2$ means that the repulsive parameter has been multiplied by 2 , etc. The coordinates referred to are: $R_{c}: C=C$ bond; $\alpha$ : $\mathrm{H}-\mathrm{C}-\mathrm{H}$ angle; $\beta$ : in-plane wag; and $\gamma$ : out-of-plane bend. $\Delta \boldsymbol{R}_{\mathrm{c}}$ denotes the deviation of the actual geometry from the reference value, $F\left(R_{c}\right)$ denotes the $M M$ force constant associated with $R_{c}$, etc. The units of the force constants are kcal mol-1. $\AA^{-2}$ for bond stretching, and $\mathrm{kcal} \mathrm{mal}^{-1} \cdot \mathrm{rad}^{-1}$ for angle bending.

the same structure and frequencies as the $a b$-initio calculation itself. The conversion procedure also provides a simple means to investigate how different parameters or functional forms of $V_{\mathrm{nq}}$ affect the $\mathrm{MM}$ force constants and the reference geometry, while the calculated frequencies and structure remain unchanged.

As a simple example the conversion has been applied to ethylene using an empirical valence force field (Pietilä et al., 1986) and nonbonded 1-4 interactions of different repulsive strengths. Some results are given in Table 1. As is seen from the table, the explicit inclusion of the nonbonded interactions has no large effect on the force constants or the reference geometry, except when the nonbonded interactions are unrealistically strong. This is of course due to the stiff valence coordinates in ethylene. However, in molecules like peptides, the effect of the nonbonded interactions is certainly much more significant. We are presently applying this approach to the conversion of an $a b$-initio force field for peptides (Cheam \& Krimm, 1990) to an MM energy function.

Acknowledgements-This research was supported by The Program in Protein Structure and Design at The University of Michigan, by a grant from the Monsanto Company, St Louis, MO, and by NSF Grant DMB-8816756.

\section{REFERENCES}

Arfken G. (1970) Mathematical Methods for Physicists. Academic Press, New York.

Cheam T. C. \& Krimm S. (1990) J. Mol. Struct. (Teochem.) 206, 173.

Fogarasi G. \& Pulay P. (1984) Annu. Rev. Phys. Chem. 35, 191.

Lii J.-H. \& Allinger N. L. (1989) J. Am. Chem. Soc. 111, 8566.

Momany F. (1978) J. Phys. Chem. 82, 592.

Pietilä L-O. (1989) J. Mol. Struct. 195, 111 (and Refs therein).

Pietilä L-O., Palmö K. \& Mannfors B. (1986) J. Mol. Spectrose. 116, I (and Refs therein). 